\title{
BMJ Open International survey on fear and childbirth experience in pregnancy and the postpartum period during the COVID-19 pandemic: study protocol
}

Kris Yuet-Wan Lok (D) , ${ }^{1}$ Rachel Wai Tung Ko, ${ }^{1}$ Heidi Sze Lok Fan, ${ }^{1}$ Janet YH Wong (D) , ${ }^{1}$ Edmond PH Choi, ${ }^{1}$ Noel Wan Man Shek, ${ }^{2}$ Hextan Y S NGAN, ${ }^{3}$ Marie Tarrant, ${ }^{4}$ Junyan Li, ${ }^{5}$ Yi-yan Huang, ${ }^{5}$ Yan-Qiong Ouyang, ${ }^{5}$ Daniel Yee Tak Fong (1) ${ }^{1}$

To cite: Lok KY-W, Ko RWT, Fan HSL, et al. International survey on fear and childbirth experience in pregnancy and the postpartum period during the COVID-19 pandemic: study protocol. BMJ Open 2021;11:e050132. doi:10.1136/ bmjopen-2021-050132

- Prepublication history for this paper is available online. To view these files, please visit the journal online (http://dx.doi. org/10.1136/bmjopen-2021050132).

Received 16 February 2021 Accepted 26 July 2021

\section{ABSTRACT}

Introduction The COVID-19 pandemic has caused unprecedented disruptions around the world. Adding to the existing stress surrounding pregnancy and childbirth, the threat of infection and social isolation policies may negatively impact pregnant women and new mothers. Literature on the effect of COVID-19 on fear during pregnancy and childbirth experience is limited. As the COVID-19 pandemic continues to affect the global population, it is important to understand how it has impacted pregnant women and new mothers' experiences worldwide to inform perinatal care and interventions. Methods and analysis This multicountry study involving China and Canada targets to recruit 1000 pregnant women and new mothers who gave birth since 2020 in each participating country. Participants will be recruited online in the local language through mothers' groups, antenatal and postnatal clinics and hospital wards. All questionnaires will be completed online. Participants' level of fear, depression and childbirth experience will be assessed along with other sociodemographic, medical and COVIDrelated measures. Regression models will be used to compare the outcomes among the participating countries. Ethics and dissemination The study has been reviewed and approved by the institutional review boards of the participating countries. Findings will be disseminated in peer-reviewed journals and academic conferences. Results from this study may guide the formulation of future health guidelines and policies in the face of a pandemic.

\section{BACKGROUND}

(C) Author(s) (or their employer(s)) 2021. Re-use permitted under CC BY-NC. No commercial re-use. See rights and permissions. Published by BMJ.

For numbered affiliations see end of article.

Correspondence to Dr Kris Yuet-Wan Lok; krislok@hku.hk
In 2019, the outbreak of the COVID-19 caused by the SARS-CoV-2 was declared a pandemic by the WHO on 11 March 2020. ${ }^{1}$ As of 16 June 2021, over 176 million confirmed cases have been recorded globally. ${ }^{2}$ The largest proportion of confirmed cases was recorded in the USA $(19.0 \%)$, followed by India $(16.8 \%)$ and Brazil $(9.9 \%))^{2}$ The case fatality rate is approximately $2 \% .^{23}$ The pandemic has caused unprecedented disruptions and
Strengths and limitations of this study

- The study will provide an international view of the impact of the COVID-19 pandemic on pregnant women and their childbirth experience.

- Measuring a large sample size in each country allows researchers to conduct subgroup analysis and cross-cultural comparisons.

- The cross-sectional design may limit the exploration of longitudinal changes within each community.

- The study results may have limited generalisability as the survey is exclusively online and selfadministered, limiting the representativeness of mothers with no internet access and low socioeconomic status.

placed an immense burden on healthcare systems worldwide.

To contain the outbreak, governments worldwide have implemented a range of public health measures, including travel restrictions, mandatory quarantine, local or national lockdowns and social distancing. ${ }^{4}$ Individuals are also advised to adopt preventive measures, avoid crowds and maintain good personal hygiene, such as frequent handwashing and wearing face coverings. ${ }^{4}$ Based on observations from previous virus outbreaks, experts have identified pregnant women as a vulnerable group and advised them to take extra precautions. ${ }^{5}$ Many hospitals have imposed limits on the number of support persons allowed in clinical visits, including antenatal and postnatal clinical visits and deliveries. ${ }^{6}$

The threat of COVID-19 infection and the abrupt changes to daily life may have an enormous impact on pregnant women and new mothers. Some studies and reviews have found that pregnant women with confirmed 
COVID-19 infection status were more likely to suffer from severe symptoms ${ }^{7}$ and experience postpartum complications. ${ }^{8}$ Other studies, however, have found no evidence of negative impact on maternal and neonatal outcomes, ${ }^{9}$ and that transmission of the virus from mothers to children is uncommon. ${ }^{9} 10$

In addition, it is likely that the mental well-being of pregnant women and new mothers to be adversely affected by restrictive public health measures and the uncertainties surrounding the pandemic. Studies have found that the COVID-19 lockdown measures have a negative impact, specifically on women. ${ }^{11}$ The proportion of pregnant women with depressive symptoms ${ }^{12-14}$ and pregnancyrelated anxiety ${ }^{121315}$ has increased since the pandemic started. As anxiety is linked to adverse maternal and neonatal outcomes, ${ }^{16}$ it is important to understand the impact of COVID-19 on fear faced during the pandemic.

The COVID-19 pandemic continues to affect societies worldwide, with some countries experiencing second or third peaks of infections and imposing new mitigation measures. Our team has commenced an international study involving 30 countries to study the impact of the pandemic on fear and health in the general population. ${ }^{17}$ As many women around the globe will have to face pregnancy, childbirth and the postpartum period under uncertain circumstances, with various government restrictions enforced in response to the pandemic. There is, thus, an urgent need for more extensive research on the impact of the pandemic on pregnancy and childbirth experience. It could provide important information with regards to the level of fear and childbirth experience of new mothers during the COVID-19 pandemic and help the development of appropriate interventions and adjustments to antenatal and postnatal care in the face of a pandemic.

To our knowledge, to date, there is no international study on the impact of the COVID-19 pandemic on fear and childbirth experience in pregnant and new mothers. In addition, how the level of pandemic severity and responses across countries and regions influence childbirth experience are yet to be examined. This study aims to address this gap and provide important information for healthcare professionals and governments.

\section{Aims}

1. To assess the level of fear among pregnant women and new mothers.

2. To assess the childbirth experience of new mothers under COVID-19 internationally.

\section{METHODS AND ANALYSIS \\ Study design}

This study will use a cross-sectional international survey, with questionnaires available in English, traditional Chinese and simplified Chinese. The survey will be available online on Qualtrics, accessible to mothers in China and Canada.

\section{Subjects and sampling}

The sample size calculation was based on an estimation of the prevalence of a health issue. We take the most conservative scenario of $50 \%$, with a $5 \%$ margin of error in a $95 \%$ CI. Therefore, we need to recruit at least 385 pregnant women and 385 new mothers. To ensure the number of responses collected is adequate after excluding incomplete questionnaires, we aim to recruit 500 participants from each group, that is, 1000 individuals, in each country.

\section{Inclusion and exclusion criteria}

Individuals are included in the study if they (1) are 18 years or older, (2) are pregnant women or new mothers who have given birth since 2020, (3) plan to or have given birth in the participating countries, (4) can read English, traditional Chinese or simplified Chinese and (5) have access to the internet. Individuals who do not meet the inclusion criteria will be excluded.

\section{Data collection procedures}

In both participating countries, a webpage has been developed to allow the completion of the questionnaires online. Individuals will be screened for eligibility on the website. Eligible individuals will be invited to participate in the study. They will be shown an information sheet on the study and be asked to provide informed consent. After signing the online consent form, participants will complete the questionnaire. Each participating country will use the same survey setup in the corresponding institution licensed Qualtrics. All these platforms will be tested by the study team and piloted with at least 10 subjects before they will be used. In addition, the survey will be promoted by snowball technique, or using social media, including Facebook, Twitter and WeChat.

Apart from the abovementioned data collection methods, potential participants will be recruited by survey service providers and promotions in mothers' groups in Hong Kong. In addition, research nurses or assistants will recruit participants in antenatal clinics or postnatal wards in public and private hospitals in Hong Kong, providing them with a link to the online questionnaire. To encourage participation, the first 500 participants in Hong Kong will be given a HK\$50 coupon after completion.

\section{Patient and public involvement}

No patient was involved.

\section{Outcome measures}

Four key outcome measures will be collected, namely, fear associated with COVID-19 infection and adverse events, level of fear, depressive symptoms and childbirth experience.

1. Fear associated with COVID-19 infection and adverse events

Participants will be asked to indicate their level of fear of their family members or themselves of infecting COVID-19, the burden of childcare provision and 
restrictions related to social distancing and childbirth. On a scale of $0-10$, a higher score means a higher level of fear, increased burden and restrictions.

\section{Fear Scale}

We adapted the eight-item Breast Cancer Fear Scale developed by Champion et al for the present study. ${ }^{18}$ The study instrument was originally developed to measure women's emotional responses to breast cancer. The Fear Scale was modified to assess the fear level on COVID-19 on eight items, each rated on a 5-point Likert scale with ' 1 ' indicating 'strongly disagree' and ' 5 ' indicating 'strongly agree'. ${ }^{18}$ A higher total score indicates a higher fear level.

3. Depressive symptoms

The Patient Health Questionnaire-9 (PHQ-9) will be administered for assessing depressive symptoms. ${ }^{19}$ It has been validated among pregnant women and is highly specific for identifying women with postpartum depression. ${ }^{20}{ }^{21}$ It comprises nine items, each rated on a 4-point Likert scale. In the scale, ' 0 ' indicates 'not at all' and ' 3 ' indicates 'nearly every day'. A higher total score of each scale indicates a higher level of depression. A translated and validated Chinese version of the PHQ-9 will be used. 223

\section{Childbirth experience}

Childbirth Experience Questionnaire 2 (CEQ2) will be used to assess participants' childbirth experience. ${ }^{24}$ CEQ2 consists of 22 statements that assess four domains of childbirth experience, that is, own capacity, professional support, perceived safety and participation. ${ }^{24}$ Nineteen items adopt a 4-point Likert scale, with ' 1 ' indicating 'totally agree' and ' 4 ' indicating 'totally disagree', while the remaining three use a visual analogue scale. Higher scores indicate a better childbirth experience. CEQ2 was originally developed in English. It has been translated into traditional and simplified Chinese.

\section{Covariates}

In addition to the four key outcome measures, the following data will also be collected:

1. Sociodemographic information, including basic demographics, perceived social status, gestational status and household size.

2. Responses to pregnancy risk assessment questions, including height, body weight and physical and psychological health history.

3. Participants' self-perception of their breastfeeding intention and current infant feeding practices.

4. Participants' self-perception of their knowledge of COVID-19; whether they themselves or someone in their immediate social circle have been infected with COVID-19, their source of information and plans for childbirth.

\section{Questionnaire}

The questionnaire will be self-administered online to measure the impact of the COVID-19 pandemic on fear and childbirth experience in pregnant and postpartum women and identify mothers' needs in times of a pandemic, appropriate preventive measures and pandemic response. The development, translation, validation and description of the questionnaire are discussed in the previous article. ${ }^{17}$

The questionnaire consisted of nine sections: (1) sociodemographic characteristics, (2) pregnancy risk assessment, (3) COVID-19 status, (4) impact of the COVID-19 pandemic, (5) COVID-19-related knowledge, (6) PHQ-9, (7) lifestyle, (8) fear and (9) childbirth experience.

\section{Planned analysis}

Data will be exported from the corresponding institution licensed Qualtrics into files of Excel and transported and cleaned in Stata. Records with incomplete responses on any outcome measurements will be discarded. For each country, descriptive statistics will be used to describe the participants' characteristics, perceptions on the health impact of COVID-19, fear, depression, anxiety, childbirth experience and other measures. Cross-country data comparisons on outcomes will be made by univariable and multivariable regression models, with weighting adjustment with age of the corresponding population..$^{25}$

All analyses will be conducted using Stata V.16, a statistical software.$^{26} \mathrm{~A} 95 \% \mathrm{CI}$ and a $\mathrm{p}<0.05$ will be considered statistically significant.

\section{DISCUSSION}

Regardless of the rising number of confirmed COVID-19 cases, our understanding of the pandemic's impact on fear and childbirth experience of pregnant women and new mothers remains limited. Our study will explore this gap on an international scale. The inclusion of both participants who are currently pregnant and those who have recently given birth would allow us to explore the impact of the pandemic on women at different stages of pregnancy and with different childbirth experiences. The results could provide important information on (1) the health-related impact of the pandemic and pandemic response on pregnant women and new mothers and (2) the unique needs of this vulnerable population group under different pandemic restrictions, hence informing intervention and healthcare policy in perinatal care. Moreover, the large sample size in each country will enable us to conduct significant subgroup analysis and may inform international and local policy and strategy on COVID-19 and future pandemic response.

Maternal mental health problems have a significant impact on women and child health. Studies show that maternal mental health problems, including antenatal depressive symptoms, ${ }^{27} 28$ anxiety ${ }^{29}$ and increased stress level, ${ }^{30}$ were associated with increased risk of perinatal complications or adverse outcomes. These problems would also affect child health outcomes. Studies show that maternal depression was associated with delayed child development ${ }^{31}$ while high-stress level during pregnancy was associated with children's emotional and behavioural problems. ${ }^{32}$ Therefore, this study would 
inform healthcare providers of the mental health needs of pregnant women and new mothers.

The international design, additionally, allows comparisons across different cultures, levels of pandemic severity and policy responses to the pandemic. As policies often change rapidly in response to the changing level of pandemic severity, results from this study could provide valuable insights for healthcare professionals to adjust the intervention and care strategies for pregnant women and new mothers accordingly. It would also strengthen future epidemic or pandemic response.

The survey website specifically developed for this study may increase the accessibility of the study. Since the website can be easily navigated on all devices and the number of smartphone users in the participating countries is high-ranging from 31.83 million to 882 million, ${ }^{33}$ accounting for about $60 \%-95 \%$ of the population-we should be able to reach our target population group effectively. Limited generalisability is one of the limitations of the study. The participants in China and Canada stated the provinces they live in, which will allow us to examine the association between the number of cases and the proposed outcome.

Despite the strengths of the study, the cross-sectional design might limit the extent to which the results might be comparable for countries at vastly different stages of pandemic or pandemic response during survey administration. Additionally, the comparability of the impact in different countries may be limited by the differences not only in the severity and public health measures but also in the healthcare system, particularly antenatal and postnatal care. With voluntary participation, the responses collected may be susceptible to self-selection bias. In addition, the survey is conducted online. Women with limited internet access and a very low level of digital literacy may not be able to participate in this study.

In summary, this study will represent an international survey on the impact of the COVID-19 pandemic on pregnant women and new mothers. It will increase our understanding of how the pandemic and various public health measures impact the fear and mental health status during pregnancy and the postpartum period, and the childbirth experience of women. Consequently, the findings will help us better understand the impact of COVID-19 in comparison to other countries in this population group. The implications of these findings will inform policymakers and healthcare professionals of the needs, enabling them to better support this vulnerable group during the pandemic locally and globally.

\section{ETHICS AND DISSEMINATION}

The study has been reviewed and approved by the Institutional Review Board of the University of Hong Kong/ Hospital Authority Hong Kong West Cluster (UW 20-490). Participation is entirely voluntary and online informed consent will be obtained from all participants. Ethical and R\&D approvals have been sought and obtained in each country involved in the study, where local ethics approval is needed. Subjects will be given information online and with informed consent through the completion of the survey. Only those who agree to participate in the study will start completing the survey. Respondents can withdraw from the study any time during the data collection process without any consequences. All information will be kept confidential, and results will be reported in aggregated form. No personal identifiable information will be collected in order to maintain the anonymity of the participants.

The research findings will be disseminated through a strategic method. The dissemination plan will include various forms of media to reach out to a wide range of stakeholder groups and individuals at the local, national and international levels. This will inevitably include the use of academic media (ie, peer-reviewed journal articles, national and international conference presentations), social media (ie, Facebook, Instagram, Twitter, LinkedIn), print media (ie, newspaper), broadcast media (ie, radio, television) and community engagement activities such as community forums or stakeholder meetings to widen our reach of dissemination.

\section{Author affiliations}

${ }^{1}$ School of Nursing, University of Hong Kong Li Ka Shing Faculty of Medicine, Hong Kong, Hong Kong

${ }^{2}$ Department of Obstetrics \& Gynaecology, Queen Mary Hospital, Hong Kong, Hong Kong

${ }^{3}$ Department of Obstetrics \& Gynaecology, University of Hong Kong Li Ka Shing Faculty of Medicine, Hong Kong, Hong Kong

${ }^{4}$ School of Nursing, The University of British Columbia, Vancouver, British Columbia, Canada

${ }^{5}$ Department of Nursing, School of Health Sciences, Wuhan University, Wuhan, Hubei, China

Contributors KYWL and RWTK drafted the manuscript. KYWL is the principal investigator of the study and is responsible for conducting the study overall. KY-WL, DYTF, EPHC, JYHW and MT conceived the study. KYWL, RWTK, HSLF, JYHW, EPHC, DYTF, MT, NWMS, HYSN contributed to the design of the study. JL, YYH, YQO and RWTK collected the data. All authors contributed to the data acquisition, critically appraised and approved the manuscript, and assume responsibility for the contents of the manuscript.

Funding The study was funded by The University of Hong Kong Start-up Fund (grant number 006027001).

Competing interests None declared.

Patient and public involvement Patients and/or the public were not involved in the design, or conduct, or reporting, or dissemination plans of this research.

Patient consent for publication Not required.

Provenance and peer review Not commissioned; externally peer reviewed.

Open access This is an open access article distributed in accordance with the Creative Commons Attribution Non Commercial (CC BY-NC 4.0) license, which permits others to distribute, remix, adapt, build upon this work noncommercially, and license their derivative works on different terms, provided the original work is properly cited, appropriate credit is given, any changes made indicated, and the use is non-commercial. See: http://creativecommons.org/ licenses/by-nc/4.0/.

\section{ORCID iDs}

Kris Yuet-Wan Lok http://orcid.org/0000-0002-3227-0799

Janet YH Wong http://orcid.org/0000-0002-3000-4577

Daniel Yee Tak Fong http://orcid.org/0000-0001-7365-9146 


\section{REFERENCES}

1 World Health Organization. WHO Director-General's opening remarks at the media briefing on COVID-19 - 11 March 2020. 2020.

2 Johns Hopkins University \& Medicine Coronavirus Resource Center. COVID-19 Dashboard 2020. Available: https://coronavirus.jhu.edu/ map.html [Accessed 16 Jun 2021].

3 World Health Organization. Estimating mortality from COVID-19: scientific brief, 2020. Available: https://www.who.int/publications/i/ item/WHO-2019-nCoV-Sci-Brief-Mortality-2020.1 [Accessed 23 Oct 2020]

4 World Health Organization. Overview of public health and social measures in the context of COVID-19: interim guidance, 2020. Available: https://www.who.int/publications/i/item/overview-ofpublic-health-and-social-measures-in-the-context-of-covid-19 [Accessed 23 Oct 2020].

5 Favre G, Pomar L, Musso D, et al. 2019-nCoV epidemic: what about pregnancies? The Lancet 2020;395:e40.

6 Rocca-Ihenacho L, Alonso C. Where do women birth during a pandemic? Changing perspectives on safe motherhood during the COVID-19 pandemic. J Glob Health Sci 2020;2.

7 Zambrano LD, Ellington S, Strid P, et al. Update: characteristics of symptomatic women of reproductive age with LaboratoryConfirmed SARS-CoV-2 infection by pregnancy status - United States, January 22-October 3, 2020. MMWR Morb Mortal Wkly Rep 2020;69:1641-7.

8 Prabhu M, Cagino K, Matthews KC, et al. Pregnancy and postpartum outcomes in a universally tested population for SARS-CoV-2 in New York City: a prospective cohort study. BJOG 2020;127:1548-56.

9 Knight M, Bunch K, Vousden N, et al. Characteristics and outcomes of pregnant women admitted to hospital with confirmed SARSCoV-2 infection in UK: national population based cohort study. BMJ 2020;369:m2107.

10 Huntley BJF, Huntley ES, Di Mascio D, et al. Rates of maternal and perinatal mortality and vertical transmission in pregnancies complicated by severe acute respiratory syndrome coronavirus 2 (SARS-Co-V-2) infection. Obstet Gynecol 2020;136:303-12.

11 Adams-Prassl A, Boneva T, Golin M. The impact of the coronavirus lockdown on mental health: evidence from the US, 2020. Available: https://www repository.cam.ac.uk/handle/1810/310906

12 Lebel C, MacKinnon A, Bagshawe M, et al. Elevated depression and anxiety symptoms among pregnant individuals during the COVID-19 pandemic. J Affect Disord 2020;277:5-13.

$13 \mathrm{Wu}$ Y, Zhang C, Liu H, et al. Perinatal depressive and anxiety symptoms of pregnant women during the coronavirus disease 2019 outbreak in China. Am J Obstet Gynecol 2020;223:240.e1-240.e9.

14 Ayaz R, Hocaoğlu M, Günay T, et al. Anxiety and depression symptoms in the same pregnant women before and during the COVID-19 pandemic. J Perinat Med 2020;48:965-70.

15 Saccone G, Florio A, Aiello F, et al. Psychological impact of coronavirus disease 2019 in pregnant women. Am J Obstet Gynecol 2020;223:293-5.
16 Dunkel Schetter C, Tanner L. Anxiety, depression and stress in pregnancy: implications for mothers, children, research, and practice. Curr Opin Psychiatry 2012;25:141-8.

17 Lok KY-W, Fong DYT, Wong JYH, et al. International survey for assessing COVID-19's impact on fear and health: study protocol. BMJ Open 2021;11:e048720.

18 Champion VL, Skinner CS, Menon U, et al. A breast cancer fear scale: psychometric development. J Health Psychol 2004;9:753-62.

19 Kroenke K, Spitzer RL, Williams JB. The PHQ-9: validity of a brief depression severity measure. J Gen Intern Med 2001;16:606-13.

20 Gjerdingen D, Crow S, McGovern P, et al. Postpartum depression screening at well-child visits: validity of a 2-question screen and the PHQ-9. Ann Fam Med 2009;7:63-70.

21 Sidebottom AC, Harrison PA, Godecker A, et al. Validation of the Patient Health Questionnaire (PHQ)-9 for prenatal depression screening. Arch Womens Ment Health 2012;15:367-74.

22 Yu X, Tam WWS, Wong PTK, et al. The patient health Questionnaire-9 for measuring depressive symptoms among the general population in Hong Kong. Compr Psychiatry 2012;53:95-102.

23 Manea L, Gilbody S, McMillan D. Optimal cut-off score for diagnosing depression with the patient health questionnaire (PHQ-9): a meta-analysis. Can Med Assoc J 2012;184:E191-6.

24 Dencker A, Taft C, Bergqvist L, et al. Childbirth experience questionnaire (CEQ): development and evaluation of a multidimensional instrument. BMC Pregnancy Childbirth 2010;10:81.

25 United Nations. World population prospects 2019: department of economic and social affairs: social dynamics, 2020. Available: https://population.un.org/wpp/DataQuery/

26 StataCorp. Stata Statistical Software: Release 16 [program]. College Station, TX StataCorp LLC; 2019.

27 Fransson E, Örtenstrand A, Hjelmstedt A. Antenatal depressive symptoms and preterm birth: a prospective study of a Swedish national sample. Birth 2011;38:10-16.

$28 \mathrm{Hu}$ R, Li Y, Zhang Z, et al. Antenatal depressive symptoms and the risk of preeclampsia or operative deliveries: a meta-analysis. PLoS One 2015;10:e0119018.

29 Glover V, O'Connor TG. Effects of antenatal stress and anxiety: implications for development and psychiatry. Br J Psychiatry 2002;180:389-91.

30 Nkansah-Amankra S, Luchok KJ, Hussey JR, et al. Effects of maternal stress on low birth weight and preterm birth outcomes across neighborhoods of South Carolina, 2000-2003. Matern Child Health J 2010;14:215-26.

31 Deave T, Heron J, Evans J, et al. The impact of maternal depression in pregnancy on early child development. BJOG 2008;115:1043-51.

32 Van den Bergh BRH, Mulder EJH, Mennes M, et al. Antenatal maternal anxiety and stress and the neurobehavioural development of the fetus and child: links and possible mechanisms. A review. Neurosci Biobehav Rev 2005;29:237-58.

33 Statista Research Department. Smartphone market in Hong Kong statistics \& facts, 2021. Available: https://www.statista.com/topics/ 5970/smartphone-market-in-hong-kong/ [Accessed 9 Feb 2021] 